\title{
Accelerating the evolution of a systolic array-based evolvable hardware system
}

\author{
Javier Mora*, Eduardo de la Torre
}

Centre of Industrial Electronics, Universidad Politécnica de Madrid, Madrid, Spain

\begin{abstract}
A B S T R A C T
Evolvable hardware is a type of hardware that is able to adapt to different problems by going through a previous training stage which uses an evolutionary algorithm to find an optimized configuration. This configuration can be achieved through dynamic partial reconfiguration of an FPGA. Having a short time for the training stage is critical for the system to be able to adapt to changing conditions in real time. However, one of the problems of evolvable hardware based on dynamic partial reconfiguration is its long evolution time, mostly due to its slow re-configuration speed. This can make such systems unsuitable for applications which require adaptation in a few seconds. Nevertheless, different reconfiguration and evolution techniques can substantially reduce the time taken by an evolvable hardware system to evolve for a specific problem.
\end{abstract}

In this article, a system initially able to evolve in 8 minutes is optimized using multiple techniques (re-configuration methodology, evolutionary algorithm optimization, and parallelization) so that it is able to obtain similar results in less than $2 s$, achieving a speedup of near 300 times. Extensive experimental results prove the benefits of such techniques.

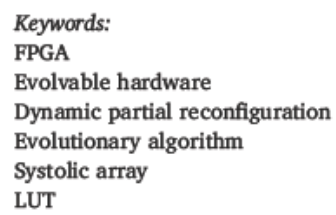

\section{Introduction}

Evolvable hardware $(\mathrm{EH})$ systems are configurable hardware systems which are able to adapt to different problems. Unlike classical system design, where the designer decides or calculates the structure and configuration of the system based on the problem specifications, EH uses an evolutionary algorithm (EA) to tune its parameters or structure in order to find the optimal configuration for a certain problem according to a set of training samples.

These training samples are representative examples of the problem that needs to be solved. For instance, a system whose purpose is to remove a certain type of noise from an image stream would use a noisy image as a training input and the same image without noise as a training reference, and a system whose purpose is to perform edge detection on an image would use a normal image as training input and the result of applying a known edge detection algorithm (which can be done in software) for the training reference. The EA would tune the hardware so that the result of processing the training input with the EH system is as similar as possible to the training reference. Once the EH has been tuned for a specific problem, it is able to process actual input for which the reference is unknown.

Obtaining a training input and reference can be done in several ways. For example, the training input could be retrieved from the actual input of the system, and then processed using a known algorithm in software in order to obtain the desired output (this process can be very slow). Once finished, the obtained image will be used as training re ference together with the training input in an EA which will tune the $\mathrm{EH}$ to emulate the work of the software. Once the $\mathrm{EH}$ has been tuned, it will be able to perform a similar task to the software but with a speed which is typically much higher. This way, the EH acts as a self adaptive hardware accelerator that mimics a software task.

The training input and reference for the case of noise removal can also be obtained by getting a generic noise free image as the training reference, and adding a specific type of noise to it in order to obtain the training input. However, this task needs knowledge of the type of noise and images that will need to be filtered. Nevertheless, previous work [1] shows that this can also be done by using the system input directly, and relying on the random nature of noise and the non locality of the filter to use two noisy inputs as training input and reference (Fig. 1).

The evolution can be extrinsic or intrinsic, depending on whether the candidate solutions are evaluated on a simulated model or on the $\mathrm{EH}$ system itself. The advantage of intrinsic evolution is that it makes the EH self healing, as it is able to recover from faults in its fabric by evolving in order to find alternative solutions where these faults have a smaller effect, making the hardware fault tolerant.

Intrinsic evolution also removes the need to use a software

\footnotetext{
* Corresponding author.

E-mail addresses javier.morad@upm.es (J. Mora), eduardo.delatorre@upm.es (E. de la Torre).
} 

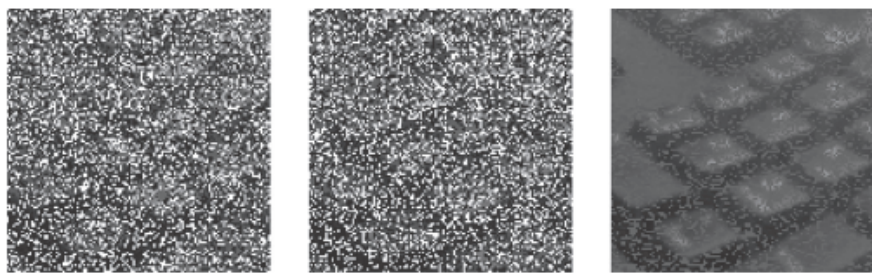

Fig. 1. Evolution without a noise-free reference. Left: training input. Center: training reference. Right: evolution result. Both input and reference have a $50 \%$ of salt and pepper noise.

simulation of the hardware in order to perform the evolution, so only the EA itself needs to be run in software. Nevertheless, the EA can be simple enough to run in an embedded processor next to the EH, or even implemented purely in a specialized hardware module; therefore the system can be implemented in a SoC, making it completely autonomous.

FPGAs are a very good platform for implementing EH, specially those equipped with dynamic partial reconfiguration (DPR) capabilities. DPR is a process through which the FPGA can autonomously re configure part of its logic while the rest continues operating. This can be used to implement an EH system as a piecewise circuit composed by multiple processing elements (PEs), each of which implements a simple task, and whose functionality can be individually changed by replacing it with a different PE using DPR.

A problem of DPR is that it is typically very slow, and is thus nor mally used only for coarse grain applications that are seldom re configured, as its intensive use in an EA would make the training times excessively long. Nevertheless, implementations that accelerate the reconfiguration by using hardware accelerated reconfiguration engines or reducing the amount of logic to be reconfigured exist $[2,3]$, leading to relatively fast reconfiguration times that take only a small fraction of the total time used by the training stage.

During the training stage, the system is either inoperative or working at suboptimal performance; therefore, it is desirable that the time taken by this stage is as short as possible. Therefore, making the training stage of DPR based EH shorter can greatly increase the amount of applications where such a system can be applied. This can be achieved through the combination of three approaches:

- Reducing the number of candidate solutions that need to be generated and evaluated by the EA until an adequate solution is found.

- Reducing the reconfiguration time needed to implement a certain solution.

- Reducing the evaluation time needed to test a certain solution once it has been configured.

The time overhead taken by the software leading the EA is typically small compared to the reconfiguration and evaluation times, specially for simple EAs.

This article describes and analyzes multiple improvements and op timization techniques that have been applied to a pre existing EH im plementation [4] in order to substantially reduce the total time needed to evolve for a particular problem, some of which were already used in [5]. By applying these techniques, the evolution time has been reduced from 8 minutes to less than 2 seconds.

The rest of the article is organized as follows: Section 2 introduces the state of the art and possible alternatives. Section 3 describes the initial implementation of the system to be optimized. Section 4 de scribes the hardware improvements made on both the hardware ar chitecture and the reconfiguration engine with the aim of reducing both reconfiguration and evaluation times, as well as reducing the resource usage of the architecture. In Section 5, certain modifications to the original EA are made in order to improve its efficiency, reducing the number of candidate solutions generated and evaluated in order to obtain good results. Section 6 takes advantage of the reduction in re source usage performed in Section 4 to parallelize the EA across mul tiple evaluation units, which further decreases the evaluation time. Finally, Section 7 shows the conclusions of the article and summarizes the improvements achieved in each section.

This article is structured in an incremental approach, where each improvement is analyzed before moving on to the next one, since most of the times an improvement is justified by the results of the previous one. There is no separate section for the results; instead, these are shown at the end of each subsection.

\section{Technical background and previous work}

Common EH based processing systems consist of a large number of basic processing units, known as processing elements (PEs), which are interconnected in a specific manner. Each of these PEs has a certain number of inputs coming from the system input or from other PEs, implements a specific operation on the data it receives from these in puts, and sends the processed result to other PEs, typically registering the result in order to create a pipelined data processing architecture. The mission of the EA is to determine which operation will be per formed by each PE and how the PEs will be interconnected; these parameters constitute a specific candidate solution.

This section describes different topologies frequently used in $\mathbf{E H}$, as well as multiple common techniques for changing its configuration.

\subsection{Interconnection topologies}

Given that allowing every PE to get its inputs from any other pos sible PE in the system would lead to excessively complex routing (which is generally bad for FPGA design) and to having excessively big mul tiplexers at the inputs of the PEs, the way in which PEs can interconnect is usually restricted so that only a few possible interconnections are allowed.

One of these interconnection topologies is the Cartesian genetic programming (CGP) [6], which consists of a series of PEs arranged in columns, as seen in Fig. 2. Each of these PEs can take data from the primary input and the columns to the left, and usually implements a stateless simple function (typically 1 bit logic gates). In order to further simplify the hardware implementation in terms of multiplexers and routing, as well as the search space for the EA, the number of inputs available to a certain PE can be constrained to a maximum number of columns to the left (typically one column, to avoid large multiplexers).

Although PEs in CGP typically implement 1 bit logic gates as their processing function [6,7], authors have shown that this is inefficient for evolvable image filters, and replace these basic logic functions with more complex functions such as $\mathbf{8}$ bit adders [8], which do not need to yield an exact result but can be simplified approximations [3]. Other authors go one step further and create complex PEs on the fly by combining primitive functions, a technique known as embedded CGP (ECGP) [9].

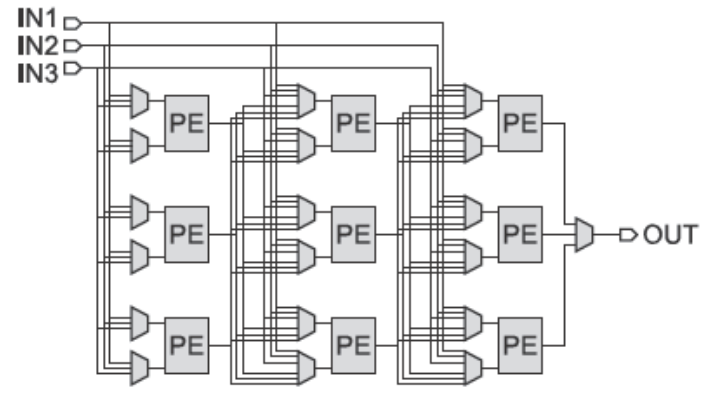

Fig. 2. Example of a $3 \times 3$ CGP topology with 3 primary inputs and 1 primary output. Each PE in this example has 1 output and 2 inputs, from either the system input or a PE in the previous column. 


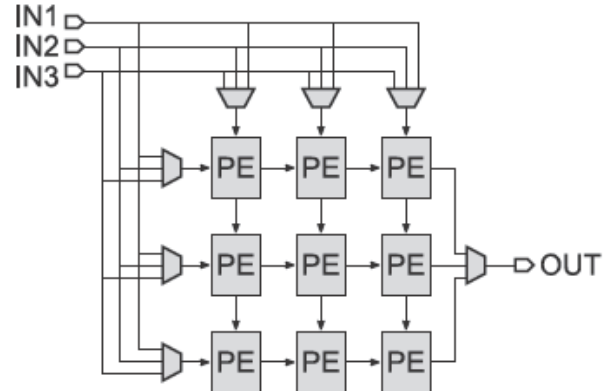

Fig. 3. Example of a $3 \times 3$ systolic array with 3 inputs and 1 output. Notice how the routing has been simplified and the multiplexers removed except for the ones at the input and output of the system.

The main problems with this topology are that the routing of the nets may still be too complex, and that the multiplexers introduce extra delay in the logic path reducing the frequency of operation, although this can be solved by registering the output of the multiplexers in ad dition to the PE outputs. Additionally, the complexity of this topology varies with scalability, as larger multiplexers are needed for bigger array sizes.

Multiplexers also have the drawback of using a high amount of re sources. For example, while an 8 bit adder processing element only needs 8 LUTs in total in modern Xilinx FPGAs, a single 13:1 multiplexer as proposed in [3] ( 9 inputs +4 PEs) requires 4 LUTs per output bit [10], 64 LUTs in total for 2 input multiplexers. Therefore, multiplexers alone would represent $89 \%$ of the resource usage for this topology.

Another topology which does not suffer this problem is the systolic array, first defined in [11] as a generic computing engine, and used as a reconfigurable fabric for implementing $\mathrm{EH}$ in [12]. It was originally intended for complex PE operations but it can be used with simpler PEs as well. Opposite to CGP, inputs to each PE are fixed, connecting each of them to its neighbors (Fig. 3).

This topology simplifies the routing between processing elements, allowing for shorter data paths and thus lower delay. Also, smaller logic resources per every PE allows PEs to be closer between neighbors. Additionally, it removes the need of having a multiplexer at the input of every PE (except at the system input), thus again reducing the delay as well as the resource usage. Its simplicity is also an advantage in dy namically scalable systems such as [4].

The disadvantage of this solution is the degradation of connectivity between PEs, which would force EAs to take longer evolution cycles to obtain correct mappings, or even limiting the maximum quality of the resulting circuits.

\subsection{Reconfiguration methods}

As said before, PEs must be able to switch between different func tions according to the configuration of a specific solution.

The most straightforward way to achieve this is by simply im plementing all possible circuits and using a multiplexer to select which function the PE uses (Fig. 4), in a similar way to how an ALU works. This is known as virtual reconfigurable circuit (VRC) [13], and has the advantage of being highly portable (independent of the FPGA used). However, this approach is considerably resource and energy

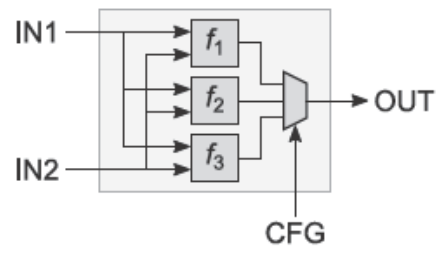

Fig. 4. VRC with 3 functions. Output may be registered (not shown).

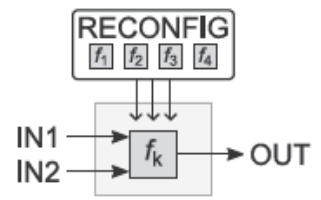

Fig. 5. By using DPR instead of VRCs, the output multiplexer can be removed and only one function is implemented, reducing the amount of resources used.

consuming, since all the possible functions have to be implemented at once, and the extra multiplexer to select the used function has the same problems CGP has: it introduces extra delay and resource usage.

This approach is used in [14] in combination with CGP in order to implement an evolvable image filter.

An alternative to VRCs consists in using DPR if the FPGA supports it. With this methodology, rather than having to implement all possible PE circuits physically in the FPGA, only one of them is present at a time. A reconfiguration engine will later be able to replace this circuit with an other one stored in a PE library by partially reconfiguring the area of the FPGA corresponding to that PE (Fig. 5).

This approach reduces considerably the amount of resources used by a single PE and potentially improves its maximum frequency of operation, at the expense of making the system specific to a certain FPGA model. Additionally, DPR introduces a reconfiguration time overhead (specially when a software driven reconfiguration engine is used, in which case the reconfiguration can take several milliseconds [15]), unlike VRCs where this time is negligible since it would only involve changing the input of some multiplexers. Nevertheless, in [2] the reconfiguration time is still small compared to the evaluation time, thanks to the usage of efficient hardware based reconfiguration en gines.

The design of modules interchangeable by means of partial re configuration requires the input and output nets of the module to be compatible with the ones on the static system. Xilinx tools provide a solution for this requirement in their partial reconfiguration flow [16]. However, this flow does not support using the same circuit on different positions (relocation) even though this is theoretically possible due to the uniformity of FPGAs.

An alternative to Xilinx's partial reconfiguration flow that allows relocation is the use of bus macros (as is done in [12]), which route nets in a replicable way, at the cost of extra delay and resource usage. More recently, the usage of bus macros has been replaced by custom tools such as Dreams [17] or GoAhead [18] which do not present this resource overhead. The Dreams tool is used in [4] to implement a scalable sys tolic array of up to $8 \times 7$ PEs, each with a size of $5 \times 1$ CLBs.

A third way to change the functionality of a circuit is to change the content of the FPGA LUTs that are used to implement logic functions, which is not as flexible as changing the complete circuit including inter connection nets, but can still be a good solution if the PEs are similar, and is easier to accomplish given that no special routing considerations have to be taken. This was done in [19] on a Xilinx Virtex II Pro by temporarily using the LUTs as shift registers in order to write a new content. However, the amount of LUTs that can work as shift registers has decreased in more modem Xilinx FPGA families, and currently only $2530 \%$ of the LUTs in Virtex 5 [20] and 7 series [10] FPGAs have this functionality, unlike Virtex II Pro which allowed this in all of its LUTs [21].

[3] uses a similar LUT reconfiguration approach in a CGP topology, but using DPR instead of relying on a shift register functionality, thus being able to use all the LUTs in the FPGA, not only the aforementioned $2530 \%$ of them. This implementation achieves a remarkable speed of 8700 candidate evaluations per second. This same approach is com bined with parallelization of multiple CGP arrays in [22] in order to obtain speeds of $\mathbf{3 0} 000$ candidate evaluations per second, completing the evolution in only $10 \mathrm{~s}$. In [5], the same approach is applied to an SA architecture, using it to reconfigure input and output multiplexers as well. 


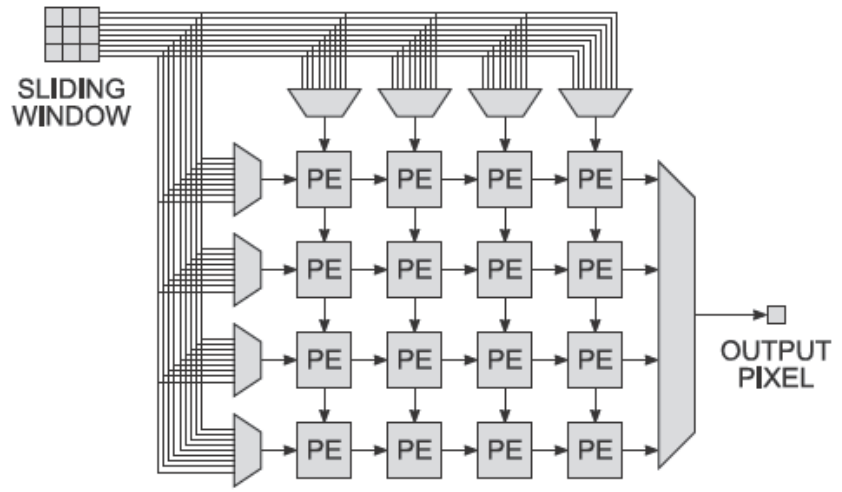

Fig. 6. Systolic array, in this case with a size of $4 \times 4$ PEs. Both outputs of each PE are actually the same signal.

\section{Initial system}

The system this work targets is an evolvable image filter based on a reconfigurable systolic array (SA), which uses dynamic partial re configuration (DPR) in order to change its functionality. The re configuration is driven by an evolutionary algorithm (EA) which runs as a software program on a MicroBlaze processor. The whole system is im plemented on a Xilinx Virtex 5 LX110T 1 FPGA.

\subsection{Hardware}

The image filter is based on a systolic array (Fig. 6) which receives data from a $128 \times 128$ pixel grayscale image by using a $3 \times 3$ pixel sliding window which moves one pixel per clock cycle to the right. The SA operates on the data of this window, generating one pixel value per clock cycle at its output. This output stream of pixels is then stored as the filtered image.

Additionally, the output is compared pixel by pixel with a reference image, using the sum of absolute errors (SAE) as a metric to measure the quality of the filter:

$\mathrm{SAE}=\sum_{i=0}^{127} \sum_{j=0}^{127}$ loutput $_{i j}-$ reference $_{i j} \mid$

This metric is used as the fitness criterion on an evolutionary algo rithm (EA) which is used to train the filter for a specific problem. This EA runs on a MicroBlaze processor.

The SA is structured as an array of processing elements (PEs) as de picted in Fig. 6. Each PE has two 8 bit inputs connected to the adjacent elements situated above ("north", $N$ ) and to its left ("west", $W$ ), and one output connected to the PEs below ("south", $S$ ) and to the right ("east", $E$ ); and implements a simple function such as identity, addition, sub traction, constant value, etc. The list of available functions is shown in Table 1. Every PE on the north and west borders of the SA takes its input from one of the 9 pixels of the input window, and the SA output is obtained from one of the PEs on the east border. The function im plemented by each of the PEs, the pixel selected at each of the inputs, and the output selected as the result constitute the configuration of the array.

This particular system was first described in [2], and relied on dy namic partial reconfiguration (DPR) of a Xilinx Virtex 5 FPGA in order to change the configuration of the processing elements, and multiplexers for the inputs and output. DPR was done using a custom hardware accelerated reconfiguration engine able to perform DMA to an external DDR2 SDRAM where the partial bitstreams containing the FPGA con figuration data are stored, rather than relying on the IP core provided by Xilinx (XPS HWICAP) which is fully software driven and lacks DMA capabilities. Additionally, although the nominal frequency of the FPGA's internal configuration access port (ICAP) is $100 \mathrm{MHz}$, this engine
Table 1

Functions implemented by the PEs.

\begin{tabular}{lll}
\hline Index & Equation & Description \\
\hline 0 & $N+W \bmod 256$ & Addition (modulo 256) \\
1 & $N+N \bmod 256$ & Multiply by 2 (modulo 256) \\
2 & $W+W \bmod 256$ & " \\
3 & $\min (N+W, 255)$ & Addition (limited at 255) \\
4 & $\min (N+N, 255)$ & Multiply by 2 (limited at 255) \\
5 & $\min (W+W, 255)$ & " \\
6 & $\left\lfloor\frac{N+W}{2}\right\rfloor$ & Average (rounded down) \\
7 & 255 & Constant value of 255 \\
8 & $\left\lfloor\frac{N}{2}\right\rfloor$ & Divide by 2 (rounded down) \\
9 & $\left\lfloor\frac{W}{2}\right\rfloor$ & " \\
10 & $N$ & Identity (pass through) \\
11 & $W$ & " \\
12 & $\max (N, W)$ & Maximum \\
13 & $\min (N, W)$ & Minimum \\
14 & $\max (N-W, 0)$ & Subtraction (limited at 0) \\
15 & $\max (W-N, 0)$ & " \\
\hline
\end{tabular}

was overclocked in order to reach frequencies of up to $250 \mathrm{MHz}$, since it was shown in [23] that overclocking the ICAP is possible.

The SA was built with PEs of a size of 20 configurable logic blocks (CLBs) in height and 2 CLBs in width (40 CLBs in total). This size was motivated by the size of a reconfiguration frame, which spans 20 CLBs vertically, not allowing the direct reconfiguration of regions smaller than this size. Additionally, routing restrictions made it difficult to implement these PEs in a size narrower than 2 CLBs. All this made it difficult to implement SAs larger than $4 \times 4$ PEs.

In order to overcome this size limitation, a new implementation was developed which had a PE size of only $5 \times 1$ CLBs [4], allowing SAs as large as $8 \times 7$ PEs. This was made possible by using a custom routing tool [17] and a feature of the reconfiguration engine which allows reading the configuration of a region with a height of one frame and write it back modifying only a fragment. However, this approach in troduced some drawbacks:

- It doubles the reconfiguration time, since it requires reading in ad dition to writing.

- The readback operation presented timing errors when overclocking the ICAP, so the frequency had to be reduced to $100 \mathrm{MHz}$.

- Even though the PE is now only 5 CLBs big, a whole column of 20 CLBs needs to be read and written in order to reconfigure a fragment of it.

Therefore, although the new PEs are smaller than the old ones, this improvement in size caused the reconfiguration time to increase.

This SA was able to operate at frequencies of $250 \mathrm{MHz}$. However, due to limitations of the logic that interfaces with it, the whole system frequency was set to $100 \mathrm{MHz}$ to prevent timing errors.

With this implementation, the system was able to generate and evaluate approximately $\mathbf{2 0 0 0}$ candidate solutions per second.

\subsection{Evolutionary algorithm}

The algorithm employed to obtain an optimal configuration is based on a simplified genetic algorithm, where each gene is an integer value that represents the function of a PE (from 0 to 15), the $3 \times 3$ sliding window pixel selected by an input selector (from 0 to 8), or the output selected by the output selector (from 0 to height 1 ). The algorithm used is a $(1+8) \mathrm{EA}$ (Algorithm 1), which is described below.

The EA starts initializing the systolic array so that all input selectors select the central pixel of the $3 \times 3$ window, all PEs copy their west input, and the output selector selects the output from the bottom right 
parameter generations $:=120000$

parameter $\lambda:=8$

$\triangleright$ total $120000 \times 8=960000$ evaluations

procedure Evolution

initialize parent

evaluate parent

for $g:=1 \ldots$ generations do

evolveGeneration parent

end for

return parent

end procedure

procedure EVOLVEGENERATION parent

for $i:=1 \ldots \lambda$ do

children $[i] \leftarrow$ parent

mutate children $[i]$

$\triangleright$ Mutation

evaluate children[i]

$\triangleright$ Evaluation

end for

bestChild $\leftarrow$ children[lowest fitness]

$\triangleright$ Selection

if fitness $($ bestChild $) \leq$ fitness (parent) then

end if

parent $\leftarrow$ bestChild

end procedure

Algorithm 1. $(1+\lambda)$-EA with $\lambda=8$.

PE, in order to obtain an identity filter. This candidate solution is evaluated by filtering a training image and comparing the filter output (currently identical to its input) with a reference image, obtaining the solution's fitness as the SAE between the obtained output and the re ference.

After initialization, an iterative process takes place. On each itera tion (generation), 8 copies (children) are made from the current config uration (parent). Each of the copies is then mutated with a mutation rate of $K=3$. This means that 3 random genes are selected and replaced by a random value. Then, the 8 mutated solutions are evaluated by re configuring the SA and filtering the training image, obtaining their fitness. Finally, the best solution among the parent and children (the one with a lowest fitness) is selected as the new parent for the next generation. In case of tie, the children have preference over the parent.

The iterative process is repeated for 120000 generations, evaluating a total of 960000 candidate solutions.

For this study, the EA has been used to obtain a filter for removing salt and pepper ${ }^{1}$ noise from an image. This was achieved by using a Lena test image (Fig. 7) to which a $20 \%$ of salt and pepper noise has been artificially added as the training image, and the original image without noise as the reference. Once the EA has concluded, the obtained filter is able to remove this type of noise from other images [24]. Pre vious works $[1,24]$ show that this kind of system evolves correctly in dependently of the training set for several types of noise, so no other images nor noise types have been tested in this work.

In order to obtain statistically meaningful results, the evolution process was repeated 1000 times with different initial random seeds. The fitness value is registered every 1500 generations, and different percentiles are then calculated for each point in the evolution. The result of this is shown in Fig. 8. As can be seen, the median of the results at the end of the evolution is approximately 40000 . As a reference, the original image has an SAE with respect to the reference of 416297, and the image filtered with a median filter using a $3 \times 3$ pixel window has an

\footnotetext{
${ }^{1}$ Salt and pepper noise consists in replacing randomly selected pixels (in this case, $20 \%$ of them) with black or white pixels.
}
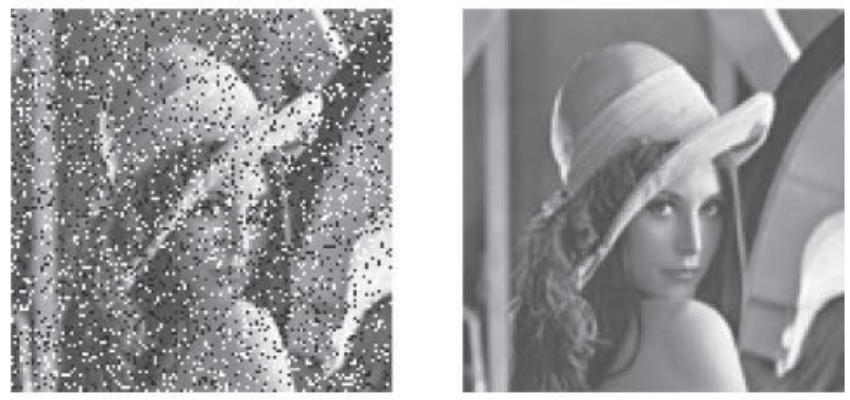

Fig. 7. Training image with $20 \%$ noise (left) and reference image without noise (right) used in the evolution.

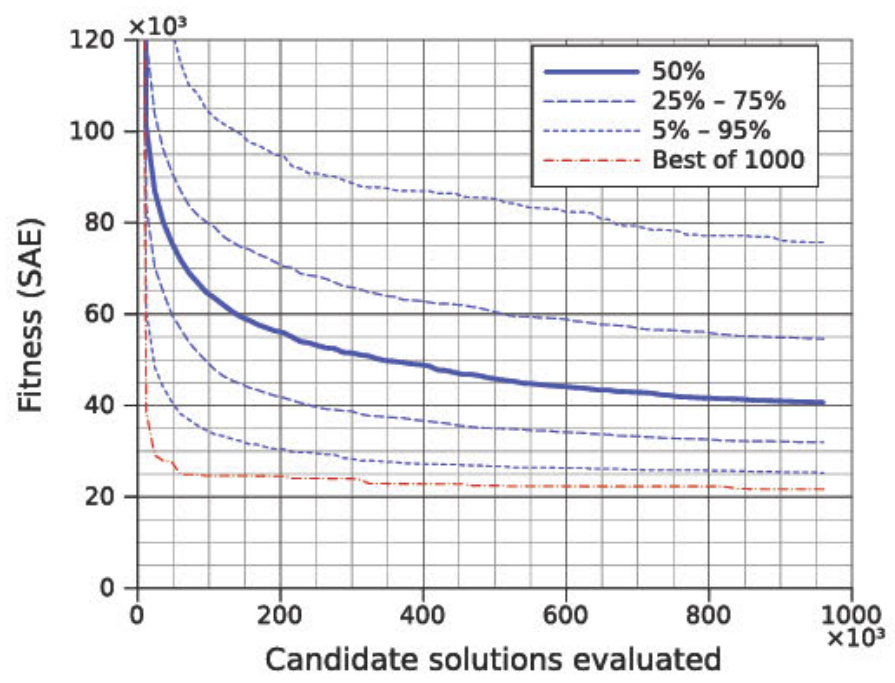

Fig. 8. Plot showing the progress of the fitness value along the evolution, displaying the median value of the 1000 evolutions (solid) as well as several confidence intervals (dashed). The best result among the 1000 evolutions is also shown. 


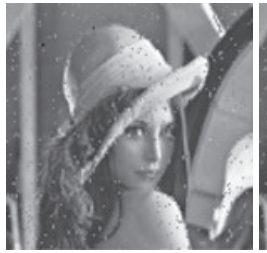

$\mathrm{SAE}=48518$

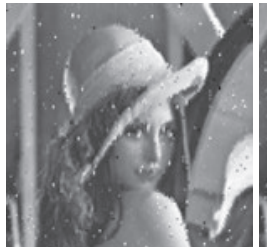

$\mathrm{SAE}=95238$

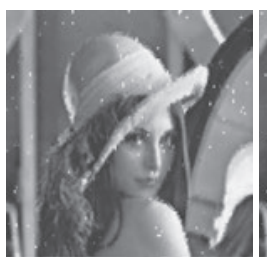

$\mathrm{SAE}=53031$

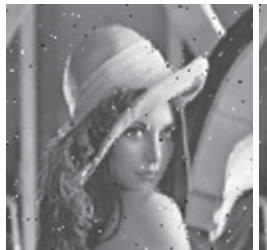

$\mathrm{SAE}=43167$

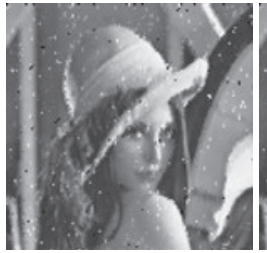

$\mathrm{SAE}=91377$

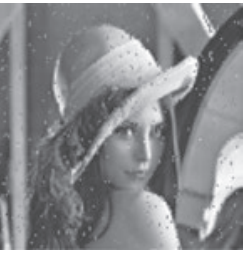

$\mathrm{SAE}=44019$

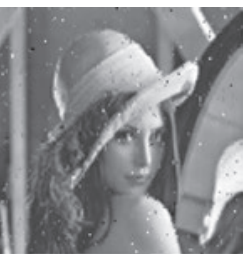

$\mathrm{SAE}=40483$

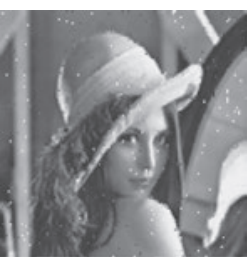

$\mathrm{SAE}=37706$

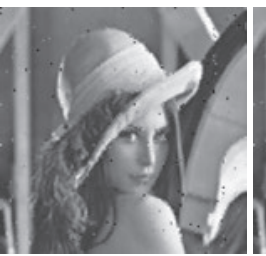

$\mathrm{SAE}=32885$

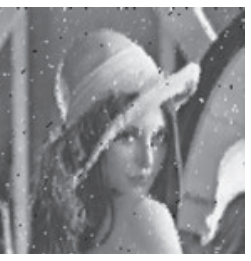

$\mathrm{SAE}=85450$

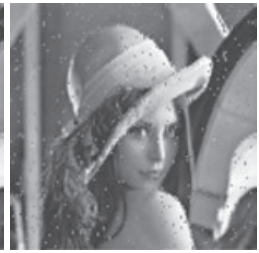

$\mathrm{SAE}=44004$

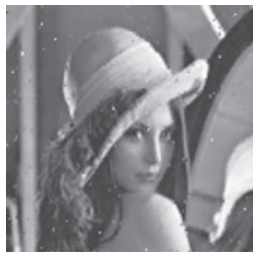

$\mathrm{SAE}=32096$

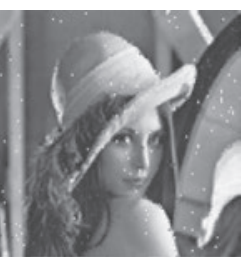

$\mathrm{SAE}=35616$

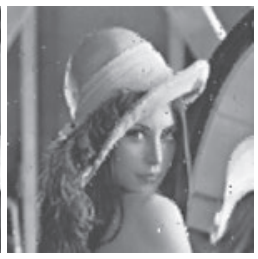

$\mathrm{SAE}=29223$

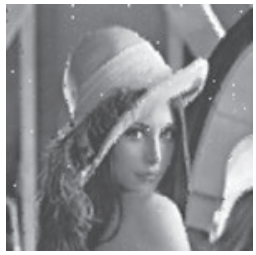

$\mathrm{SAE}=31298$
Fig. 9. Some of the results obtained during different runs of the evolution, after 60000 (left), 240000 (middle), and 960000 (right) candidate solution evaluations (7500, 30000 , and 120000 generations).

SAE of 84187 , which is outperformed more than $95 \%$ of the times. The SA used for these tests has a size of $8 \times 8$ PEs.

Fig. 9 shows some of the results obtained at the end of the evolution for different evolution lengths.

\subsection{Evaluation of results and conclusions}

The implementation described in [4] is able to evaluate 2000 can didate solutions per second. The current EA requires 960000 evalua tions to complete (although a shorter evolution with fewer evaluations can still yield acceptable results). Therefore, the time required to evolve is $480 \mathrm{~s}$ (8 $\mathrm{min}$ ). This long evolution time limits the amount of appli cations for this system; for example, it may be unsuited for adaptive real time applications requiring very fast adaptations.

The evolution time could be reduced by simply reducing the number of generations performed by the EA, but this would affect the quality of the obtained filter. Therefore, the system needs to be optimized in order to achieve a considerably shorter evolution time.
The current EA shows a quick progress at the beginning, but this progress is slowed down at the end. This may be because this kind of EA tends to get stuck at local optima, and once this happens it will typically take a long time to find a better solution. Therefore, finding strategies to avoid local optima or minimize their effect may improve the efficiency of the EA.

In the next sections, several modifications are applied to this system in order to reduce the evolution time.

\section{Hardware improvements}

\subsection{Reconfiguration engine improvement}

In order to overcome the drawbacks of the reconfiguration engine described in Section 3.1, a new engine targeting fine grain re configuration has been designed. The main difference with the former one is that, rather than relying on ICAP readback for the modification of a fragment of a whole CLB column, the new engine simply rewrites all the PEs of the column, leaving to the software the task of memorizing which PEs were on that column before. Therefore, the software will only have to pass a list of memory addresses where the partial bit streams of the respective PEs are stored.

In order to access the partial bitstream data quickly, the external DDR2 SDRAM has been replaced with an internal block RAM (BRAM), which can be accessed in arbitrary order at one word per clock cycle. This type of memory is much more limited in size than the external DDR2 SDRAM; however it is enough for fine grain reconfiguration ap plications, given the small size of the PEs, which can be stored in as little as $128 \mathrm{~KB}$.

The reconfiguration engine has been simplified and optimized, and operates at a speed of $200 \mathbf{~ M H z}$. This, together with the removal of the readback stage, have made the reconfiguration time 4 times shorter.

\subsection{PE optimization: LUT reconfiguration}

Another optimization of the hardware system has involved re designing the PEs so that they all have the same structure, differing only on the logic configuration. This removes the need of reconfiguring the interconnections, needing only to reconfigure the lookup tables (LUTs) that implement the logic functions of the circuit, which amounts to only 8 of the 36 configuration frames needed to reconfigure a whole CLB column in the Virtex 5 family, thus making the reconfiguration time considerably shorter. Additionally, it removes the need of special routing considerations, which allows implementing the whole PE in a size as small as $2 \times 1$ CLBs, needing only 128 CLBs to implement an SA of $8 \times 8$ PEs.

This new version of the PEs was developed in collaboration with Prof. Roland Dobai from the Brno University of Technology. The structure of these PEs is shown in Fig. 10, and is implemented in 16 LUTs ( 2 per each output bit), 8 FFs and two 4 bit carry chains, all of which fits on 4 Virtex 5 slices. By providing the right LUT configuration, this PE structure can implement all of the functions in Table 1. In order to further reduce the amount of logic to reconfigure, unused LUT inputs are tied to zero; this reduces the number of frames to reconfigure to 3 (1 for the first LUT and 2 for the second one) rather than all 8 of them.

The input selectors on the north and west borders of the SA are implemented in a similar way (Fig. 11) and changed using DPR as well, rather than using actual multiplexers which would require additional logic for changing their control registers. In order to simplify the system, only 3 of the 9 pixels from the input window are fed to each input selector; the remaining 6 are obtained by delaying this input by a varying amount of clock cycles. Input selectors are pipelined in order to be synchronous with the pipelining of the PEs.

The output selector is built by cascading LUTs as shown in Fig. 12, and is pipelined as well. This way of pipelining the PEs, inputs, and output is done in order to synchronize the circuit so that data from 


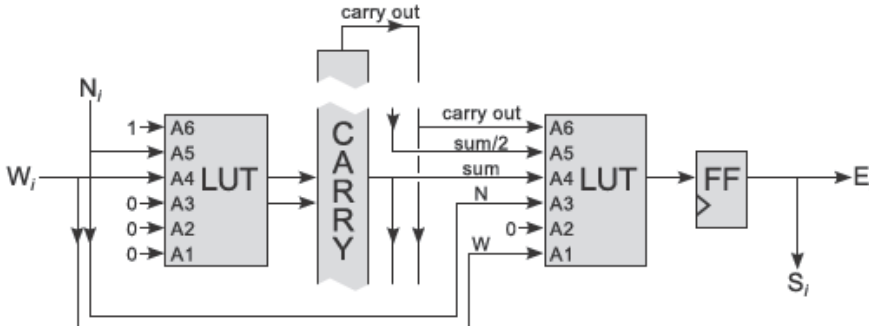

Fig. 10. Fragment of a PE. Each PE is constituted by 8 fragments like this, 1 per bit. The sum/2 signal is the sum signal (extended with the carry out bit) shifted 1 bit to the right.
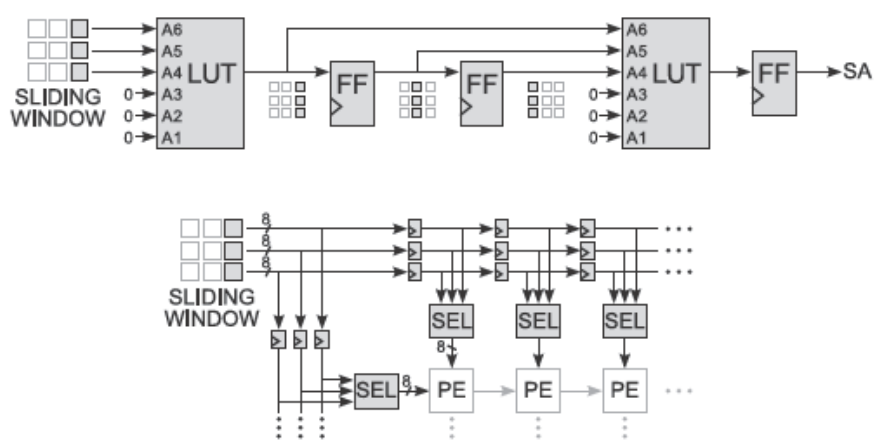

Fig. 11. Top: fragment of an input selector; each input selector is constituted by 8 fragments like this. Bottom: pipelining of the input selectors.

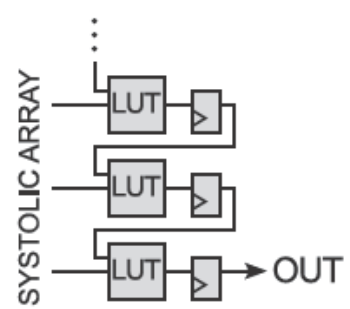

Fig. 12. Diagram of the output selector (for 1 of the 8 bits). The size of this selector depends on the height of the array.

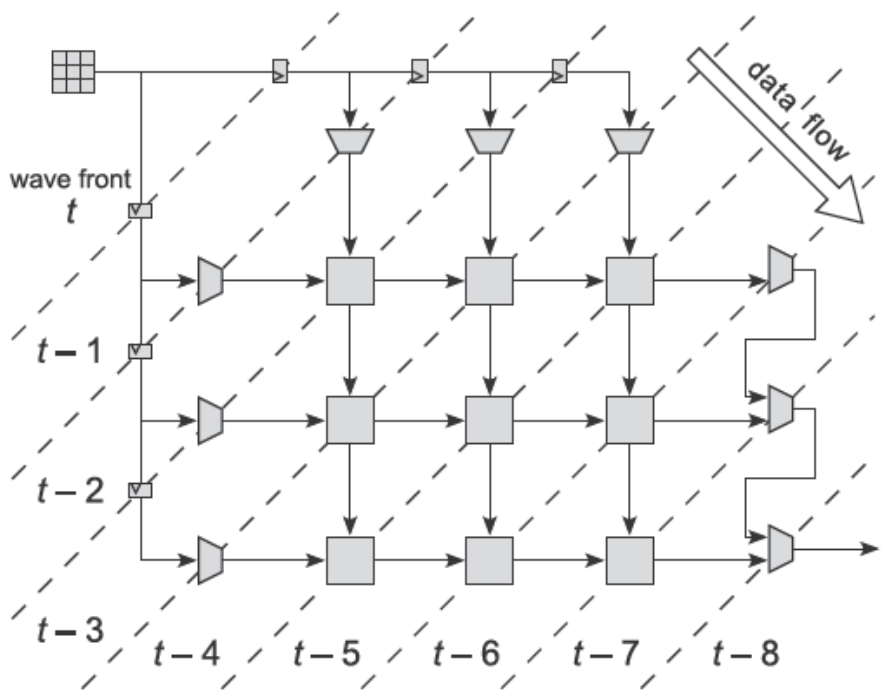

Fig. 13. Data propagation through the SA. Due to the way elements are registered, data propagates in separate wave fronts. The values on each wave front depend exclusively on the input window at a certain clock cycle, and the SA output at time $t$ is a function of the input at time $t-8$ in this example. input windows at different clock cycles does not mix (Fig. 13) and its behavior is functionally equivalent to a purely combinatorial one.

The reduced size of the new PEs (only 2 CLBs) allows for a very compact implementation, resulting in short interconnections that pro vide a good timing behavior. This makes the new SA implementation able to operate at $400 \mathrm{MHz}$, which is close to the $600 \mathrm{MHz}$ theoretical maximum clock frequency achievable on this FPGA [25].

However, some parts of the logic interfacing with it such as the comparator (SAE computing unit) cannot reach this speed. In order to overcome this limitation, the system has been divided into multiple clock domains, using dual clock asymmetric FIFOs to synchronize the data, as shown in Fig. 14. This allows having the SA work at $400 \mathrm{MHz}$ while the BRAMs and the comparator work at $100 \mathrm{MHz}$, processing 4 pixels in parallel in order to keep the throughput at 400 megapixels per second.

Additionally, the reduced size of the PEs and the fact that only a few frames need to be reconfigured reduces the required size of the BRAM to less than $2 \mathrm{~KB}$, making it able to fit in a single RAMB36 block (which has a capacity of $4 \mathrm{~KB}$ ).

\subsection{Evaluation of results and conclusions}

By redesigning the PE structure so that all its configuration relies on LUT content and implementing an improved and optimized DPR engine that targets fine grain DPR, the reconfiguration times have been re duced considerably. Additionally, the design has become much more compact, which makes it resource efficient and able to operate at high frequencies.

With these improvements, the new implementation has increased the evaluation speed from 2000 to 19000 evaluations per second, making the evolution time almost 10 times shorter. This hardware is able to perform the same evolution process in $\mathbf{5 0} \mathrm{s}$.

The reduction of the $\mathrm{PE}$ size and the removal of routing restrictions via the use of LUT reconfiguration allow implementing SAs of a larger size (tested to sizes up to $24 \times 24$ ) or putting multiple arrays working in parallel.

The advantage of using DPR to reconfigure LUTs over other tech niques such as using LUTRAM or SRL is that it allows maximizing the local occupancy, i.e., using $100 \%$ of the LUTs on the FPGA region used by the SA, rather than being restricted to a small number of them.

\section{EA improvements}

Another way to tackle the problem of having a long evolution time is to optimize the EA so that it reaches the solution faster, needing fewer evaluations in order to obtain the same result. In this section, different evolution strategies are studied and compared.

\subsection{Mutation rate}

Previous work made in this research line has typically used muta tion rates of $K=3$. This mutation rate was chosen experimentally by performing a few tests on a $4 \times 4 \mathrm{SA}$, and it was observed that $K=3$ yielded good enough results. However, as the array size increases, it becomes important to analyze the mutation rate to see if improvements can be obtained by varying it. Additionally, it should be noted that larger mutation rates involve performing a larger number of re configurations, increasing the reconfiguration time and thus slowing down the evolution. Therefore, it would be interesting to make the mutation rate as small as possible without affecting the obtained quality.

Fig. 15 shows the results of the EA with different mutation rates, performing 1000 runs for each of them. Since the EA is still rather slow and a high number of tests are involved, the EA has been run for only 30000 generations (240 000 evaluations).

As can be seen, the best results are obtained for mutation rates of 2 


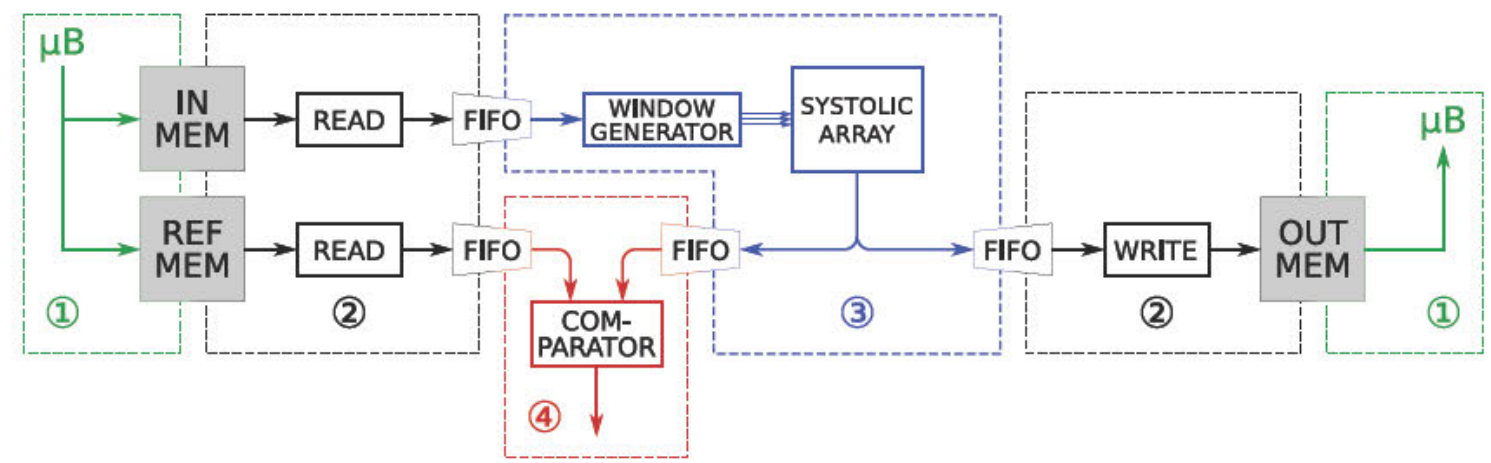

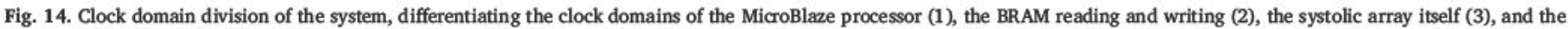
comparator (4), which is able to process multiple pixels in parallel.
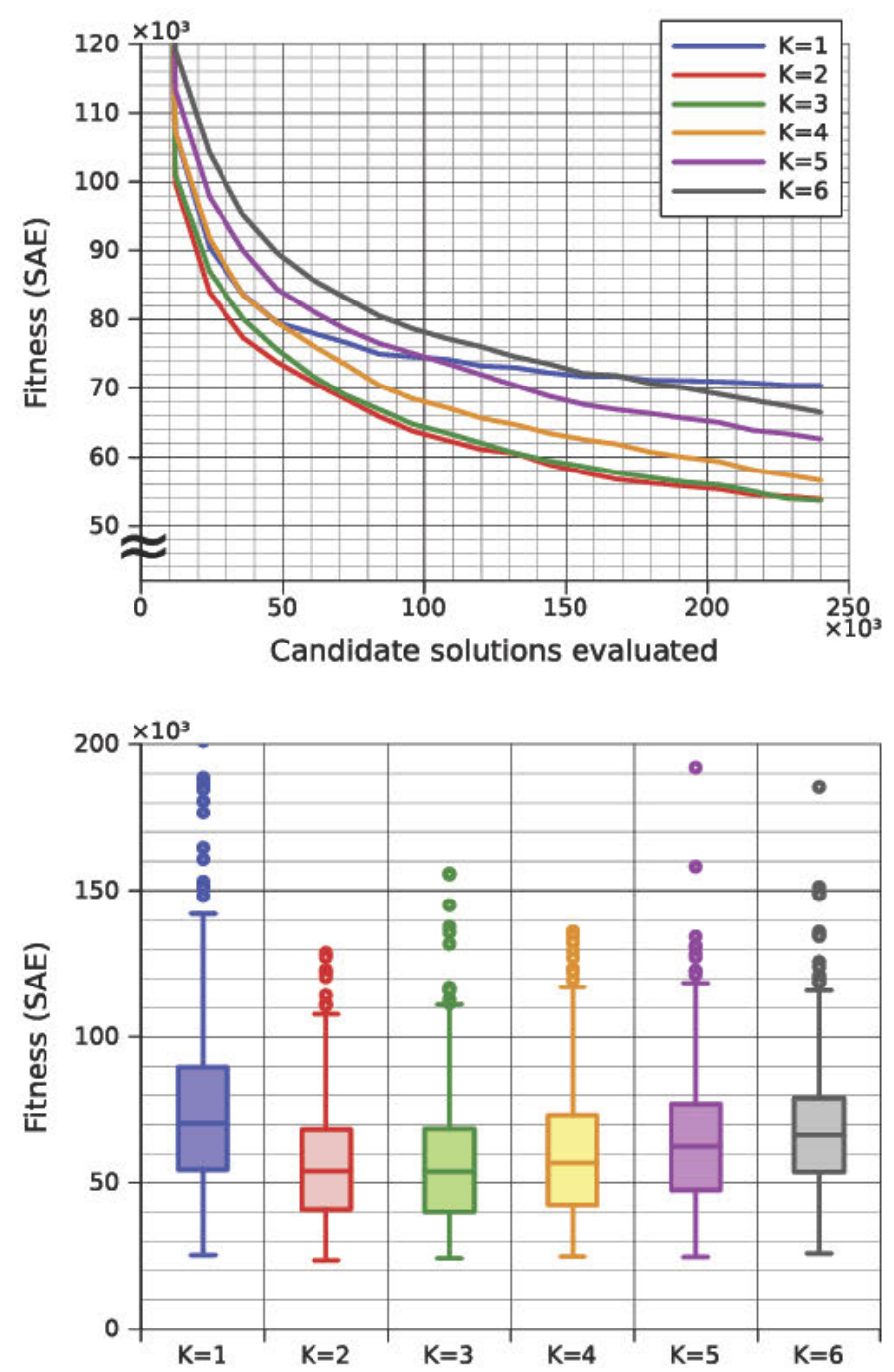

Fig. 15. Results obtained for different mutation rates, showing the median value of the fitness (top) and the fitness distribution after evolving for a total number of 240000 evaluations (bottom).

and 3, with nearly identical results. A mutation rate of 1 gives very poor results, causing the evolution to rapidly stall. Mutation rates of 4 or more make the evolution converge more slowly, and thus yield worse results for a same evolution time. Additionally, as it has been stated, high mutation rates cause a longer reconfiguration time. Therefore, given that values of 2 and 3 produce the best results, a mutation rate of 2 becomes a more reasonable choice.

In order to ensure that the difference between these results is statistically meaningful and not a result of an excessively small sample size (even for a size of 1000 values), a two tailed Student's $t$ test has been performed. This test yielded a $p$ value of 0.92 for $K=2$ versus $K=3$, not indicating statistical significance, and less than $10^{-16}$ for $K=2$ versus any other value, indicating that the apparent difference seen in Fig. 15 is statistically significant.

This applies to SAs with $8 \times 8$ PEs; larger sizes would probably require greater mutation rates, so separate experiments should be made for said sizes.

\subsection{Offspring size}

The described $(1+8) \mathrm{EA}$ is a particular case of the more general $(1+\lambda) \mathrm{EA}$, where $\lambda$ is the offspring size, this is, the number of children tested in each generation. This is typically done in evolution strategies, which are EAs based on real numbers where a small change typically causes an improvement or regression with similar probabilities. However this is not the case for the EA used here, which is closer to a genetic algorithm.

Fig. 16 compares the $(1+8) \mathrm{EA}$ with a $(1+1) \mathrm{EA}$, for the same number of evaluations (not generations), both with $K=2$. As can be seen, both algorithms yield very similar results. This suggests that the number of children does not affect the outcome of the EA for the same number of evaluations.

Given this result, the $(1+1)$ EA has been chosen over the $(1+8)$ EA in order to simplify its implementation and further modifications. Nevertheless, both strategies are equally valid, so an $(1+8)$ EA (or any

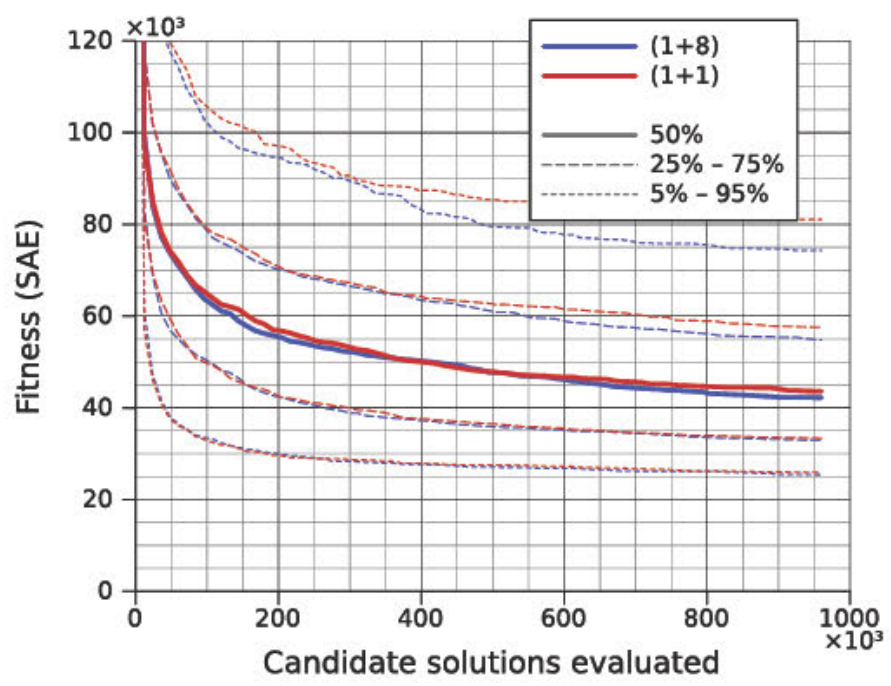

Fig. 16. Results for a $(1+8)$-EA run for 120000 generations and a $(1+1)$-EA run for 960000 generations. Both algorithms perform the same number of evaluations $(960000)$, and thus take the same time to complete. 


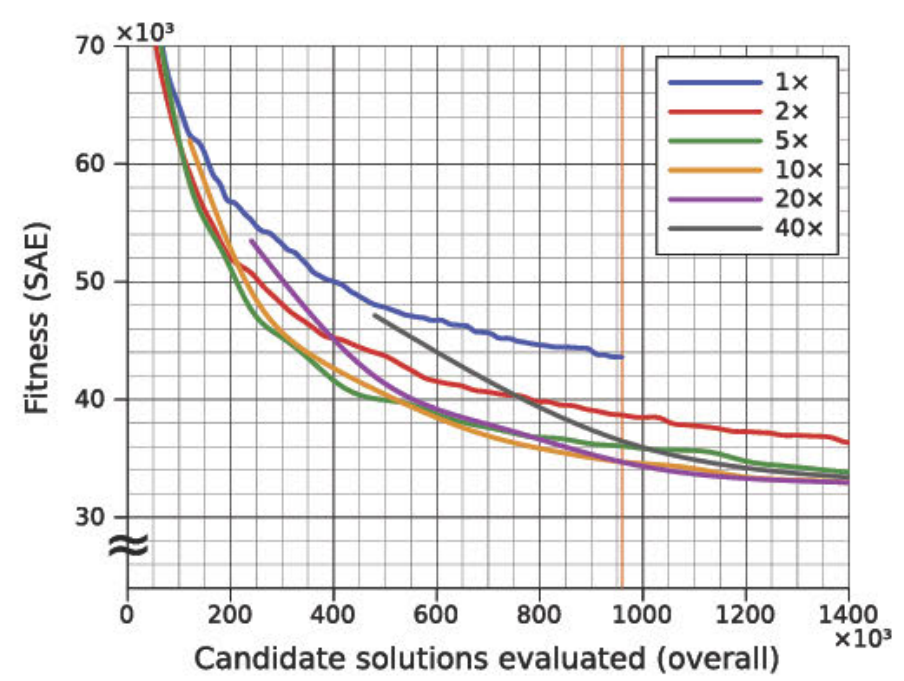

Fig. 17. Fitness of the best of $n$ evolutions (median). The $\mathrm{X}$ axis represents the combined number of evaluations for the $n$ evolutions, and is therefore proportional to the time required to perform the whole training. The vertical orange line marks the 960000 evaluation limit.

other $(1+\lambda)$ EA) could be used if needed (for example to exploit par allelizability over multiple SAs, as is done in [22]).

\subsection{Increasing the diversity}

The main problem of the original EA is that it is prone to getting stuck at a local optimum. This causes that the EA barely improves after a certain number of generations.

Fig. 8 showed that the dispersion of the fitness results at the end of the evolution is rather high, with their median value being at 40000 but $90 \%$ of the results ranging from 25000 and 75000 . The curves near the end have a very reduced slope; therefore the evolution could be made shorter without this having a great impact on the result.

An easy way to obtain better results is to repeat the evolution multiple times, and pick the best result. For example, although the probability of getting a result below 32000 (25th percentile) is only $25 \%$, the probability of having any of 5 results below that value is $76 \%$. This involves running the EA multiple times, but since the gain towards the end of the evolution is small, it would be possible to shorten each of the evolutionary runs.

Fig. 17 shows the median fitness of the best of $n(1+1)$ EA evolu tions for a certain number of evaluations. This value has been estimated from the data obtained for a single $(1+1) \mathrm{EA}$ using the formula $f_{n}(p)=f_{1}(1-\sqrt[n]{1-p})$ with $p=0.5$, where $f_{1}(p)$ is the quantile function of the fitness for a single $(1+1)$ EA (the value for which there is a probability $p$ of obtaining a fitness lower than that value), and $f_{n}(p)$ is the quantile function for the "best of $n$ " approach.

As can be seen in Fig. 17, performing 25 proportionally shorter evolutionary runs yields better results than performing a single one. A higher number of evolutionary runs shows a worse performance for a small number of evaluations since excessively short individual evolu tions tend to yield bad results; however, this changes as more evalua tions are made. Specifically, for 960000 evaluations, the optimum is in 1020 evolutions.

Fig. 18 shows the experimental results of selecting the best of 12 $(1+1)$ EA evolutionary runs of 80000 generations each. The number 12 was chosen because it is between 10 and 20 , which show the best results in Fig. 17, and for reasons that will be justified in Section 6. As can be seen, the new strategy outperforms the old one after 150000 evaluations, and can obtain results similar to the old one in half as many evaluations; additionally, the dispersion of these results is smaller.

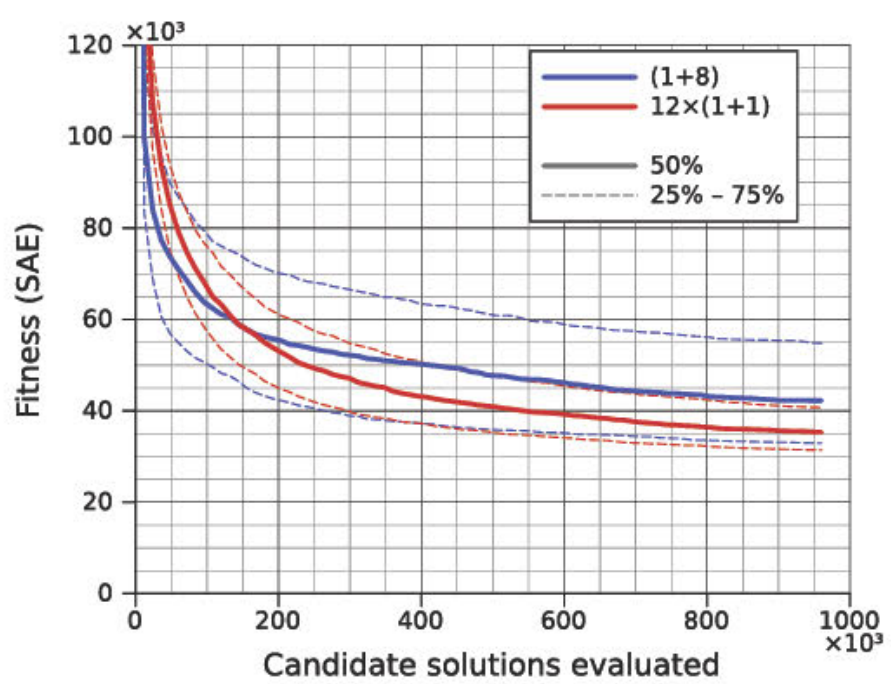

Fig. 18. Comparison of the original $(1+8)$-EA with 120000 generations with the best of $12(1+1)$-EA with 80000 generations. Both strategies evaluate a total of 960000 candidate solutions.

In addition to obtaining better results, the new strategy is easily parallelizable, being able to distribute the evolutionary runs across multiple SAs which can even be on different devices; it can also be executed on a single device with a single SA by sequencing all the evolutionary runs, memorizing the final result of each one.

\subsection{Promoting good evolutions}

Although the strategy described in Section 5.3 reduces the effect of the EA getting stuck at local optima, there is still a risk that some of the 12 evolutionary runs get stuck, becoming of no use for the rest of the evolution. Being able to remove these evolutionary runs and giving advantage to the rest would improve the result.

A way to achieve this is to execute all the evolutionary runs con currently, performing an exchange every 1000 generations. In each exchange, the evolution with the worst fitness is considered to be likely stuck and is terminated, and the one with the best fitness is considered as a promising evolution and is forked in order to improve the chances of success (Algorithm 2).

As can be seen in Fig. 19, this modification further improves the results. The new strategy is able to achieve the results of the original one in less than 240000 evaluations. Therefore, the evolution can be made 4 times shorter while still obtaining similar results.

\subsection{Restricting mutations to a single column}

Finally, a way to reduce the time spent in reconfiguration is to force all the mutations to be on the same column of the SA. Since the re configuration engine described in Section 4.1 writes the PE configura tion in whole columns, forcing the mutations to be in the same column reduces the number of columns to reconfigure, thus decreasing the reconfiguration time by approximately a $30 \%$.

As can be seen in Fig. 20, the effect of this simplification on the fitness is negligible.

\subsection{Evaluation of results and conclusions}

By optimizing the EA, the number of evaluations (and therefore the overall evolution time) can be reduced 4 times while still achieving similar results. This reduces the total evolution time to less than $12 \mathrm{~s}$.

By reducing the mutation rate and restricting all mutations to a single column, the reconfiguration time has been reduced. Currently, the reconfiguration time is small compared to the time spent filtering 


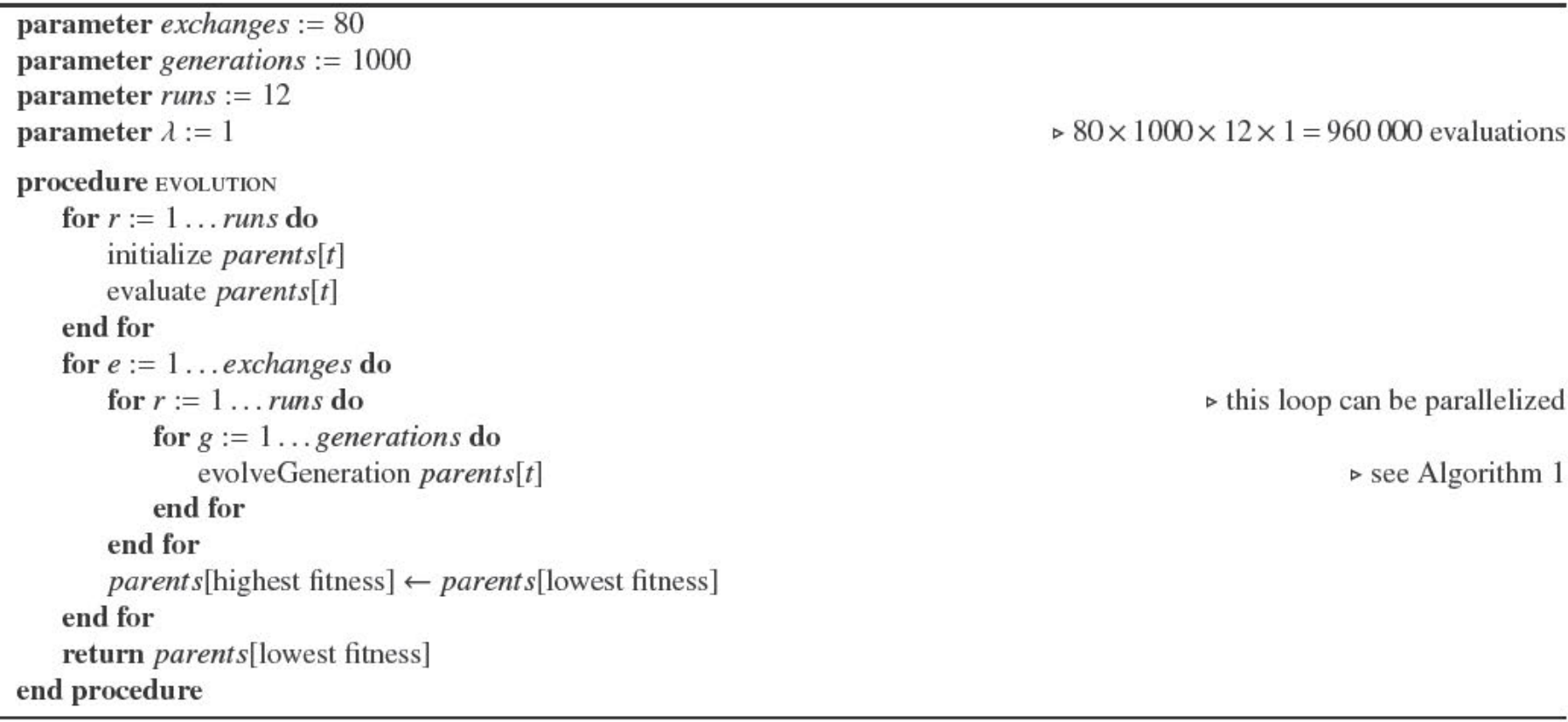

Algorithm 2. $12 \times(1+1)$-EA with exchanges.

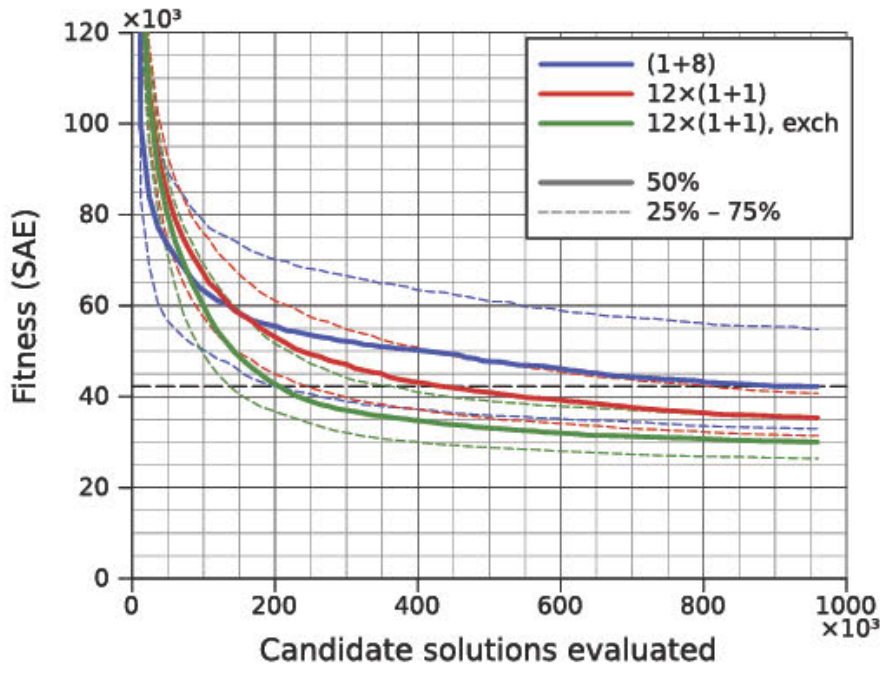

Fig. 19. Comparison of the original $(1+8)$-EA, the best of $12(1+1)-\mathrm{EA}$, and $12(1+1)$ EA with an exchange every 1000 generations (12 000 evaluations), all with $K=2$. The horizontal dashed line marks the median fitness obtained with the original $(1+8)$-EA with $K=2$ (42 346).

the image, as will be seen in Section 6, so these savings are not too beneficial for now; nevertheless they will have a greater weight once the improvements in said section have been applied.

\section{Parallelization}

After the optimizations described in Section 4, the time spent on the different stages required to obtain a single candidate solution are shown in Table 2. "Mutation" refers to the time spent by the software gen erating the specification of a new configuration; "reconfiguration" is the time used by the reconfiguration engine writing said specification on the FPGA fabric; "filtering" is the time spent filtering the training image with the new configuration, and "selection" is the software time used to decide if the newly obtained configuration is to be kept or if it

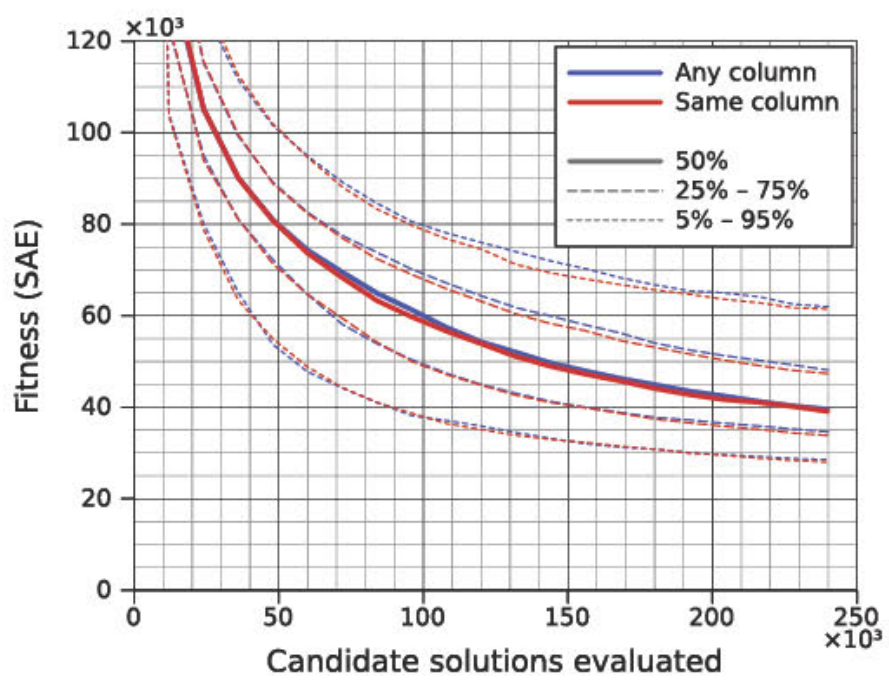

Fig. 20. $12 \times(1+1)$-EA with exchanges, with $K=2$, comparing the results of mutating PEs and selectors in any column and restricting them to a single column.

Table 2

Evolution time breakdown in microseconds for a single evaluation.

\begin{tabular}{lll}
\hline Stage & Time $(\mu s)$ & \\
\hline Mutation & 1.5 & $(3.1 \%)$ \\
Reconfiguration & 5.2 & $(10.6 \%)$ \\
Filtering & 42.0 & $(85.7 \%)$ \\
Selection & 0.3 & $(0.6 \%)$ \\
Total & 49.0 & \\
\hline
\end{tabular}

has to be discarded.

As can be seen, the majority of the time is used by the filtering stage, so reducing this time would greatly improve the overall evolution time.

As a side note, the time spent by the filtering stage is very close to the theoretical time required by the hardware $(41.3 \mu \mathrm{s})$. However, the 


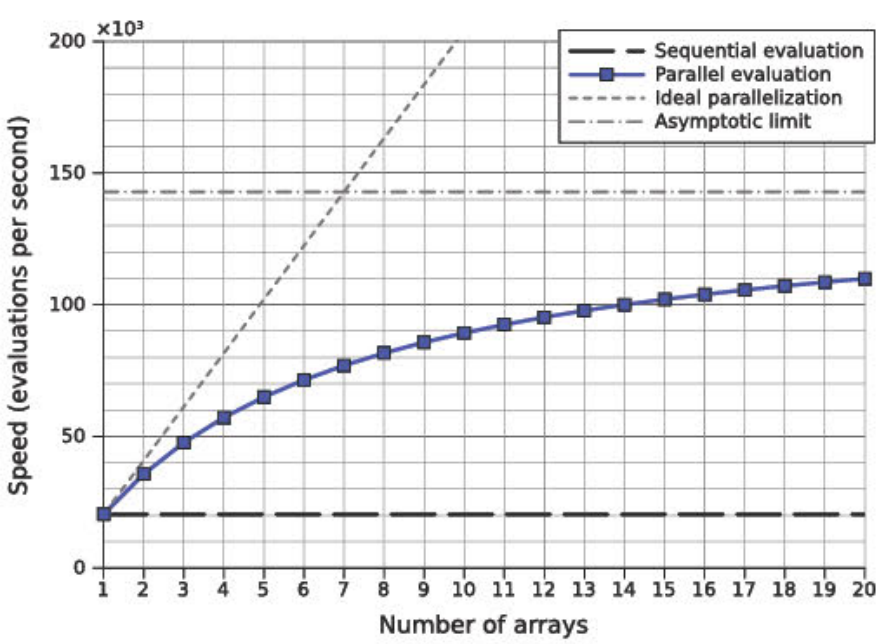

Fig. 21. Number of evaluations per second achieved with multiple SAs filtering in parallel, compared with using a single SA at a time and an ideal "totally parallelized" model in which all stages are parallelized (this could be achieved using multiple FPGAs).

theoretical time for reconfiguring has been estimated to be less than $2.8 \mu \mathrm{s}$, near half the time observed in practice. This is due to the overhead introduced by the software when driving the reconfiguration engine.

\subsection{Multiple arrays in parallel}

The systolic array already operates at a frequency close to the switching limit of the FPGA, so it is unlikely that further optimizations would bring a great improvement in the evolution times. However, the reduction in the resource usage of the SA achieved by using smaller PEs, together with the removal of the routing restrictions, allows im plementing multiple SAs which may work in parallel, thus accelerating the filtering stage.

However, this acceleration only benefits the time spent filtering the image, not the mutation, reconfiguration, and selection times, which are not parallelized since they run in software and only one ICAP can be used at a time. Therefore, the number of candidate solutions evaluated per second does not grow proportionally with the number of SAs, as can be seen in Fig. 21; it grows asymptotically to a limit equivalent to having a filtering time of 0 but keeping the other three times.

This technique is used in [5] with 8 SAs working in parallel, where speeds of 81000 evaluations per second were achieved. Using more arrays would involve a great increase in the resource usage for a small performance gain: having twice as many arrays would only improve the speed by a $27 \%$.

\subsection{Scheduling}

The previous subsection does not consider the fact that all the SAs are idle while a single one of them is being reconfigured, and that the microprocessor and reconfiguration engine are idle while the SAs are filtering. However, the current implementation does not allow launching an image filtering before the previous one has finished, since the access to the input image is done through a single memory port.

Nevertheless, it would be possible to distribute the SAs into two groups, as seen in Fig. 22, so that while one of the groups is filtering the image, the other one is being mutated and reconfigured. With enough SAs, this is equivalent to eliminating the filtering time, since it will run in the background while the other stages are completed.

The effect of this is illustrated in Fig. 23, which shows that, although this strategy involves underutilizing the SAs, the speed gain is linear rather than asymptotic, overtaking the previous approach for 6 or more SAs. The maximum speed is reached at 12 SAs. Above that point, mutation and reconfiguration times become the limiting factors, so no gain is obtained at all with more SAs.

The removal of the routing restrictions in Section 4 simplifies the placement of SAs on arbitrary positions of the FPGA, which combined

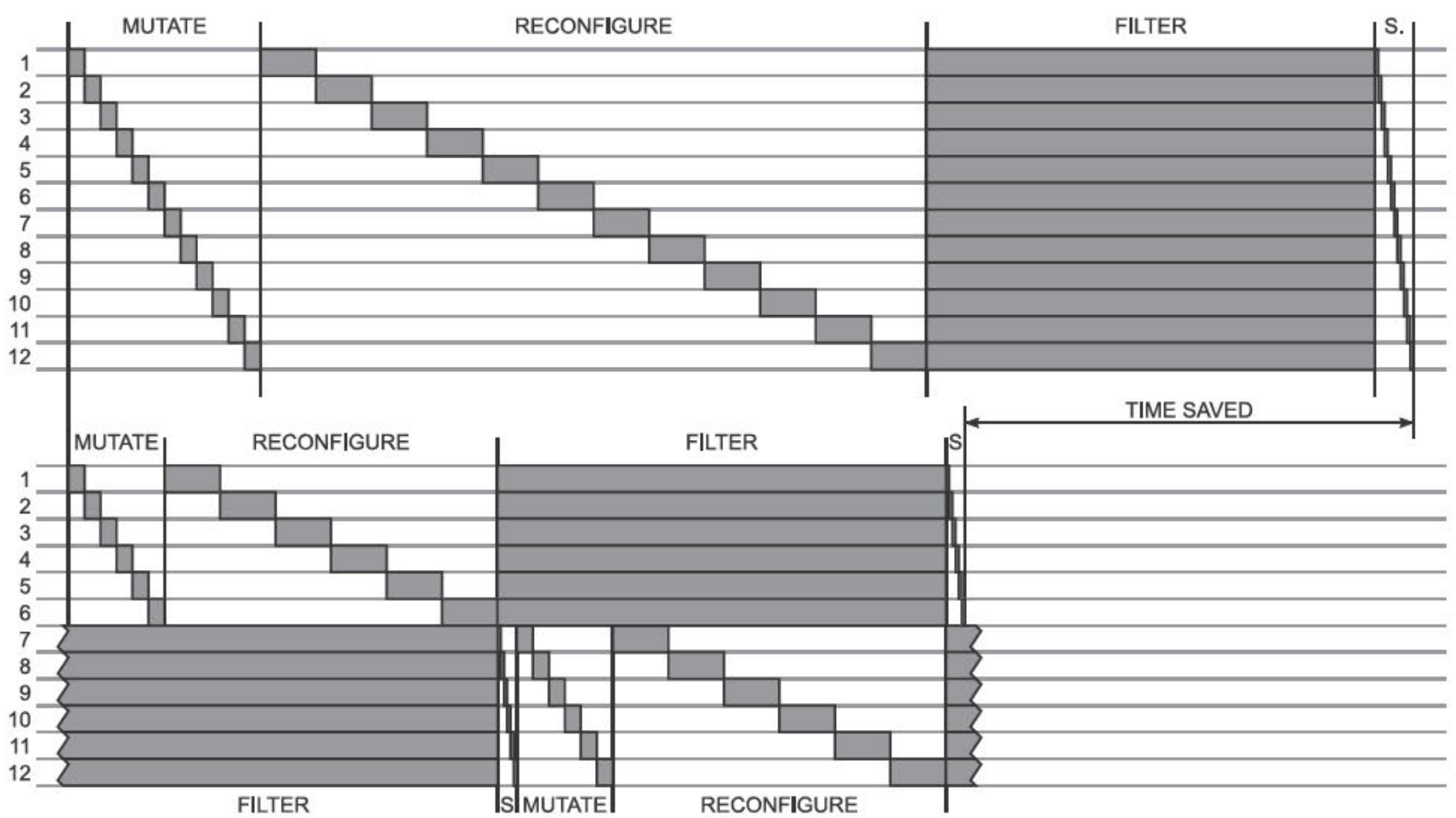

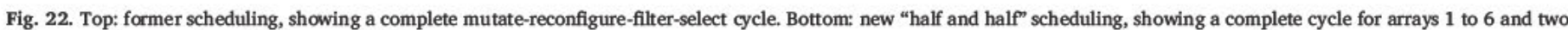
fragments of consecutive cycles for arrays 7-12. 


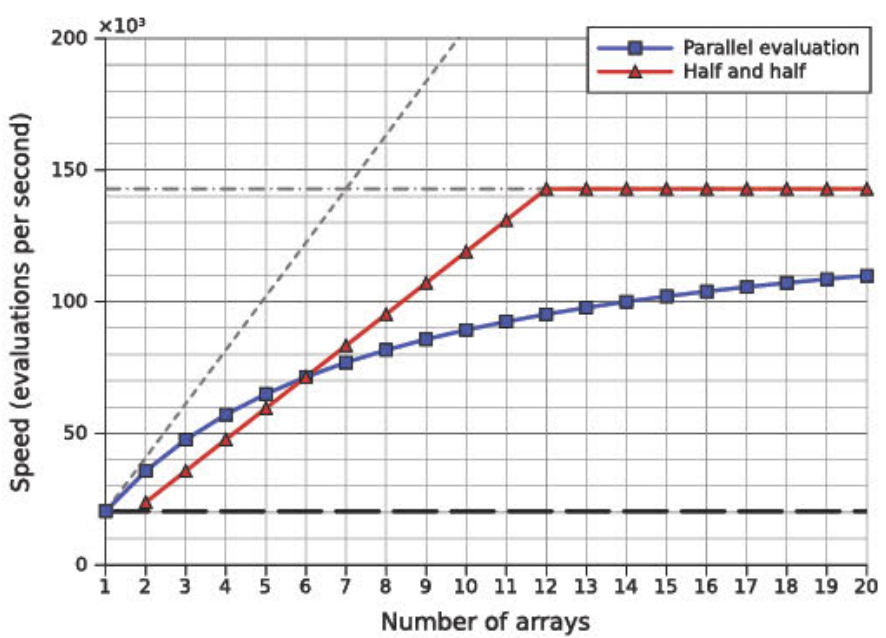

Fig. 23. Comparison of the previous parallelization approach with the half-and-half scheduling.
Table 3

Contribution of each of the improvements to the evolution speedup.

\begin{tabular}{|c|c|c|c|c|c|c|}
\hline \multirow[t]{2}{*}{ Section } & \multicolumn{3}{|c|}{ Time $(\mu \mathrm{s})$} & \multirow[t]{2}{*}{ Evals } & \multirow[t]{2}{*}{ Total (s) } & \multirow[t]{2}{*}{ Accel } \\
\hline & Ref & Filt & SW & & & \\
\hline $\begin{array}{l}\text { Initial system } \\
\text { Hardware }\end{array}$ & 333 & 166 & 2.5 & $960 \mathrm{k}$ & 481 & \\
\hline Reconf. engine & 74 & 166 & 2.5 & $960 \mathrm{k}$ & 233 & $\times 2.1$ \\
\hline LUT reconf. & 9.3 & 42 & 2.5 & $960 \mathrm{k}$ & 51.6 & $\times 4.5$ \\
\hline $\begin{array}{l}\text { Section total } \\
\text { EA }\end{array}$ & & & & & & $\times 9.3$ \\
\hline Mutation rate & 7.5 & 42 & 1.8 & $960 \mathrm{k}$ & 49.2 & $\times 1.05$ \\
\hline Offspring size & 7.4 & 42 & 1.8 & $960 \mathrm{k}$ & 49.1 & $\times 1$ \\
\hline Best of 12 & 7.4 & 42 & 1.8 & 480k & 24.6 & $\times 2$ \\
\hline Exchanges & 7.4 & 42 & 1.8 & 240k & 12.3 & $\times 2$ \\
\hline Single column & 5.2 & 42 & 1.8 & $240 \mathrm{k}$ & 11.8 & $\times 1.05$ \\
\hline $\begin{array}{l}\text { Section total } \\
\text { Parallelization }\end{array}$ & & & & & & $\times 4.4$ \\
\hline 12 parallel SAs & 5.2 & 3.5 & 1.8 & $240 \mathrm{k}$ & 2.52 & $\times 4.6$ \\
\hline Half-and-half & 5.2 & (0) & 2.0 & $240 \mathrm{k}$ & 1.73 & $\times 1.5$ \\
\hline Section total & & & & & & $\times 6.8$ \\
\hline Total & & & & & & $\times 279$ \\
\hline
\end{tabular}

with the reduction of the PE size has allowed implementing a system with 12 arrays in parallel (Fig. 24). This system is able to reach speeds of 139000 evaluations per second, a $71 \%$ more than the 81000 described in [5]. Notice that without the half and half scheduling de scribed here, a system with 12 SAs would only be able to perform 95000 evaluations per second.

Notice that this parallelization approach is not compatible with al gorithms such as a single $(1+12) \mathrm{EA}$, which requires completing the 12 evaluations in a generation before starting the next generation; there fore the first half of a generation would not be able to start until the second half of the previous one has concluded. This is not a problem for the current $12 \times(1+1) \mathrm{EA}$, since the 12 concurrent evolutionary runs can be carried out independently, only having to wait to each other every 1000 generations.

\subsection{Evaluation of results and conclusions}

Although parallelization does not provide a linear increase in the evaluation speed, a great speedup can be obtained by implementing up to 12 arrays in parallel and using half of them while the other half is reconfigured. This accelerates the evolution 7 times with respect to having a single SA. This has led to an evolution speed of 139000 evaluations per second, the fastest achieved so far, to the best of our knowledge.

For more than $12 \mathrm{SAs}$, mutation and reconfiguration times become the limiting factors, so the current approach cannot be further opti mized unless these times are reduced. Both mutation and reconfigura tion are limited by the microprocessor, so using a hardware im plementation of the EA that communicates directly with the reconfiguration engine would reduce this time. If this time were re duced to the theoretical minimum required by the reconfiguration en gine (2.8 $\mu \mathrm{s})$, speeds of up to 350000 evaluations per second could be theoretically achieved by using $30 \mathrm{SAs}$ in parallel; however these many SAs would occupy approximately $70 \%$ of the resources of the FPGA currently in use (Xilinx Virtex 5 LX110T), so it is possible that such an implementation requires a larger FPGA.

The implementation of 12 arrays in parallel has motivated the specific choice of 12 concurrent evolutionary runs in Section 5; this way, each one can be carried on independently on one SA, simplifying the implementation. Nevertheless, different numbers of concurrent evolutionary runs could be implemented, such as 24 (by performing 12 first and 12 later) or 6 (by using a $(1+2)$ EA in order to occupy the 12 SAs on each generation). This would allow further tuning of the EA to obtain maximum efficiency, but this is out of the scope of this article. 


\section{Conclusions}

In this article, multiple optimizations applied to an evolvable hardware system in order to accelerate its evolution have been de scribed. Combining the improvements in hardware, EA, and paralleli zation techniques, a total speedup of the evolution of $\mathbf{2 8 0}$ times has been achieved, going from 480 seconds to only $1.73 \mathrm{~s}$ and achieving speeds of 139000 evaluations per second. Table 3 summarizes the individual contribution of each improvement to the reduction in evo lution time.

As can be seen, the biggest improvement in terms of time comes from the improvement of the reconfiguration engine and the SA opti mization. The optimization of the EA also provides an important speedup. These two changes are independent, since the former sig nifies a reduction in the time used in each evaluation whereas the latter reduces the overall number of evaluations. The parallelization im provement also adds an important boost to the system performance, although in this case it is dependent on the other two improvements: it comes at the cost of a higher resource usage, which has been possible thanks to the reduction in SA resource usage; and relies in the reduction of reconfiguration times and EA parallelizability.

This article demonstrates that, although achieving a high evaluation speed is important in order to reduce evolution times, an efficient EA can also mean a great speedup, with the additional advantage that the latter does not involve performing changes in the hardware. Therefore it would be worth considering to analyze the current EA for a finer parameter tuning (number of concurrent evolutionary runs, interval between exchanges, variable mutation rates...) or to implement other evolution techniques such as crossover.

It should be noted that, although this work has been done on a Virtex 5 FPGA, most of its results are relevant to other platforms: LUT based reconfiguration and array parallelization can be performed on newer FPGAs such as the 7 series (and are already done by some au thors [22]), and the EA improvements are independent of the platform.

\section{Acknowledgments}

This work was partially supported by the Spanish Ministry of Economy and Competitiveness under the project REBECCA [reference TEC2014 58036 C4 2 R], and the FPI grant program of the aforemen tioned Ministry [grant number BES 2012 060459]. The LUT based version of the PEs was developed in collaboration with Prof. Roland Dobai from the Brno University of Technology.

\section{References}

[1] J. Mora, Á. Gallego, A. Otero, E. de la Torre, T. Riesgo, Noise-agnostic adaptive image filtering without training references on an evolvable hardware platform, Design and Architectures for Signal and Image Processing (DASIP), 2013 Conference on, (2013), pp. 182-189.

[2] A. Otero, R. Salvador, J. Mora, E. de la Torre, T. Riesgo, L. Sekanina, A fast reconfigurable 2D HW core architecture on FPGAs for evolvable self-adaptive systems, Adaptive Hardware and Systems (AHS), 2011 NASA/ESA Conference on, (2011), pp. 336-343, http://dx.doi.org/10.1109/AHS.2011.5963956.

[3] R. Dobai, L. Sekanina, Low-level flexible architecture with hybrid reconfiguration for evolvable hardware, ACM Trans. Reconfigurable Technol. Syst. 8 (3) (2015) 20:1-20:24, http://dx.doi.org/10.1145/2700414.

[4] Á. Gallego, J. Mora, A. Otero, E. de la Torre, T. Riesgo, A scalable evolvable hardware processing array, 2013 International Conference on Reconfigurable Computing and FPGAs (ReConFig), (2013), pp. 1-7, http://dx.doi.org/10.1109/ ReConFig.2013.6732266.

[5] J. Mora, A. Otero, E. de la Torre, T. Riesgo, Fast and compact evolvable systolic arrays on dynamically reconfigurable FPGAs, Reconfigurable Communication-centric Systems-on-Chip (ReCoSoC), 2015 10th International Symposium on, (2015), pp. 1-7, http://dx.doi.org/10.1109/ReCoSoC.2015.7238087.

[6] J.F. Miller, An empirical study of the efficiency of learning boolean functions using a cartesian genetic programming approach, in: W. Banzhaf, J. Daida, A.E. Eiben, M.H. Garzon, V. Honavar, M. Jakiela, R.E. Smith (Eds.), Proceedings of the Genetic and Evolutionary Computation Conference, 2 Morgan Kaufmann, Orlando, Florida, USA, 1999, pp. 1135-1142.

[7] L. Sekanina, Z. Vasicek, Approximate circuit design by means of evolvable hardware, Evolvable Systems (ICES), 2013 IEEE International Conference on, (2013), pp. 21-28, http://dx.doi.org/10.1109/ICES.2013.6613278.

[8] L. Sekanina, Image filter design with evolvable hardware, Lect. Notes Comput. Sci 2002 (2279) (2002) 255-266. http://www.fit.vutbr.cz/research/view pub.php? id $=6889$

[9] K. Glette, T. Gruber, P. Kaufmann, J. Torresen, B. Sick, M. Platzner, Comparing evolvable hardware to conventional classifiers for electromyographic prosthetic hand control, Adaptive Hardware and Systems, 2008. AHS '08. NASA/ESA Conference on, (2008), pp. 32-39, http://dx.doi.org/10.1109/AHS.2008.12.

[10] Xilinx, Inc. 7 Series FPGAs Configurable Logic Block (UG474), 2014.

[11] H. Kung, C. Leiserson, Systolic Arrays for (VLSI), CMU-CS, Carnegie-Mellon University, Department of Computer Science, 1978.

[12] R. Salvador, A. Otero, J. Mora, E. de la Torre, T. Riesgo, L. Sekanina, Evolvable 2D computing matrix model for intrinsic evolution in commercial FPGAs with native reconfiguration support, Adaptive Hardware and Systems (AHS), 2011 NASA/ESA Conference on, (2011), pp. 184-191, http://dx.doi.org/10.1109/AHS.2011. 5963934.

[13] L. Sekanina, Virtual reconfigurable circuits for real-world applications of evolvable hardware, Lect. Notes Comput. Sci. 2003 (2606) (2003) 186-197. http://www.fit. vutbr.cz/research/view pub.php?id $=7150$

[14] Z. Vašíček, L. Sekanina, An evolvable hardware system in Xilinx Virtex II Pro FPGA, Int. J. Innovative Comput. Appl. 1 (1) (2007) 63-73, http://dx.doi.org/10.1504/ IJICA.2007.013402.

[15] K. Glette, P. Kaufmann, Lookup table partial reconfiguration for an evolvable hardware classifier system, 2014 IEEE Congress on Evolutionary Computation (CEC), (2014), pp. 1706-1713, http://dx.doi.org/10.1109/CEC.2014.6900503.

[16] Xilinx, Inc. Partial Reconfiguration User Guide (UG702), 2013.

[17] A. Otero, E. de la Torre, T. Riesgo, Dreams: A tool for the design of dynamically reconfigurable embedded and modular systems, 2012 International Conference on Reconfigurable Computing and FPGAs, (2012), pp. 1-8, http://dx.doi.org/10. 1109/ReConFig.2012.6416740.

[18] C. Beckhoff, D. Koch, J. Torresen, Go Ahead: A partial reconfiguration framework, Field-Programmable Custom Computing Machines (FCCM), 2012 IEEE 20th Annual International Symposium on, (2012), pp. 37-44, http://dx.doi.org/10.1109/FCCM. 2012.17.

[19] K. Glette, J. Torresen, M. Hovin, Intermediate level FPGA reconfiguration for an online EHW pattern recognition system, Adaptive Hardware and Systems, 2009. AHS 2009. NASA/ESA Conference on, (2009), pp. 19-26, http://dx.doi.org/10. 1109/AHS.2009.46.

[20] Xilinx, Inc. Virtex-5 FPGA User Guide (UG190), 2012.

[21] Xilinx, Inc. Virtex-II Pro and Virtex-II Pro-X Platform FPGAs: Complete Data Sheet (DS083), 2011.

[22] R. Dobai, K. Glette, J. Torresen, L. Sekanina, Evolutionary digital circuit design with fast candidate solution establishment in field programmable gate arrays, Evolvable Systems (ICES), 2014 IEEE International Conference on, (2014), pp. 85-92, http:// dx.doi.org/10.1109/ICES.2014.7008726.

[23] C. Claus, R. Ahmed, F. Altenried, W. Stechele, Towards Rapid Dynamic Partial Reconfiguration in Video-Based Driver Assistance Systems, Springer Berlin Heidelberg, Berlin, Heidelberg, pp. 55-67. doi:10.1007/978-3-642-12133-3 8.

[24] R. Salvador, A. Otero, J. Mora, E. de la Torre, T. Riesgo, L. Sekanina, Self-reconfigurable evolvable hardware system for adaptive image processing, IEEE Trans. Comput. 62 (8) (2013) 1481-1493, http://dx.doi.org/10.1109/TC.2013.78.

[25] Xilinx, Inc. Virtex-5 FPGA Data Sheet: DC and Switching Characteristics, 2016

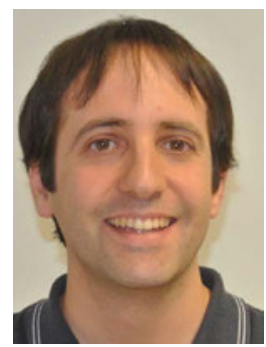

Javier Mora received the BSc degree in Industrial Engineering from the Universidad Politécnica de Madrid (UPM), Spain, in 2011, and the MSc in Industrial Electronics in 2013 from the same University. He is currently working toward the $\mathrm{PhD}$ degree in Industrial Electronics at UPM. He has been a grant-holding researcher at the Centre of Industrial Electronics (CEI), UPM, and currently holds an FPI grant from the Spanish Ministry of Economy and Competitiveness. His current research area is in the field of evolvable hardware and dynamic partial reconfiguration of FPGAs.

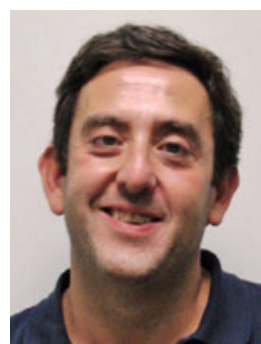

Eduardo de la Torre is Associate Professor in Electronics at the Universidad Politécnica de Madrid (UPM), Spain, doing his research at the Centre of Industrial Electronics. He obtained his PhD in Electrical Engineering from UPM in 2000 His main expertise is in FPGA design, embedded systems design, signal processing and partial and dynamic reconfiguration of digital systems. He has participated in nine EU funded projects and, overall, in six funded projects related with reconfigurable systems. 